\title{
Les médecins, trop nombreux ou pas assez?
}

L

e grand débat canadien sur les soins de santé occupe l'avant-scène de la campagne électorale fédérale en

cours et personne ne s'en étonne. L'insatisfaction face aux listes d'attente, aux services d'urgence, à la sécurité des patients et à l'accès aux soins primaires attise la ferveur alors que les partis se bousculent pour la première place avec de grandes promesses comme les soins à domicile, l'assurance-médicaments et le financement fédéral d'appoint, et des engagements aussi ciblés que «l'augmentation des places réservées à la profession médicale dans les universités et la reconnaissance accélérée des titres étrangers des nouveaux immigrants ${ }^{1} \gg$.

Promettre plus de médecins, ce n'est pas très risqué sur le plan politique : rares sont les électeurs qui diront qu'il y en a déjà assez. Il est toutefois difficile de prédire les besoins futurs en professionnels de la santé : beaucoup diraient même que c'est impossible. Au Canada, nous avons connu le rapport Barer-Stoddart de $1991^{2}$ (dont une version longue a été publiée dans le $7 A M C$ dans une série d'articles à partir de 1992) ${ }^{3}$ dont les auteurs prédisaient un surplus de médecins et qui a entraîné une réduction de l'inscription dans les facultés de médecine et du nombre de postes de formation postdoctorale.

Nous avons publié plus récemment des prévisions de pénuries de médecins ${ }^{4}$ - ainsi que des prévisions contraires prévoyant des surplus de médecins ${ }^{5}$.

On peut fonder les prévisions sur la rétrospective ou la prospective. Celles qui reposent sur des statistiques historiques supposent que les tendances du passé se maintiendront. Ainsi, pour déterminer le besoin de médecins dans 10 ans, il suffit de se fonder sur les projections existantes de la croissance future de l'économie, qui sont reliées très étroitement au nombre de médecins. Aux Etats-Unis, des projections indiquent qu'il manquera 50000 médecins d'ici à $2010^{6}$. Selon ces mêmes prévisions, on aura aussi besoin de beaucoup plus de spécialistes que de médecins de famille.

Par ailleurs, on peut prédire la demande future en imaginant un système idéal de soins de santé et en s'y préparant. $M$. Romanow a suivi cette démarche pour établir ses projections des effectifs dans son rapport final' ${ }^{7}$. Sa méthode suppose avec raison que si les professionnels de la santé travaillaient en équipe, chaque profession utilisant son propre éventail de compétences spécialisées (champ d'exercice professionnel) de façon complémentaire et avec un soutien administratif et technologique adéquat, nous n'aurions probablement pas besoin de plus de médecins. Le problème de ce modèle, c'est bien entendu que pour le faire fonctionner, il faut instaurer des ô changements fondamentaux dans la culture des soins de santé et de la formation des professionnels - des changements qui, en dépit de décennies d'efforts, continuent de nous échapper.

Comme il est impossible de prévoir combien de professionnels de la santé (et de quelles disciplines) on aura besoin dans 10 ans, il est tentant de se contenter de continuer de s'en sortir tant bien que mal - ce qui n'est toutefois pas vu comme une politique publique respectable. Les associations de profession- nels de la santé, les universités et les provinces (qui ont compétence et budgets en matière d'éducation) n'auront d'autre choix que d'adopter une orientation stratégique claire fondée sur des estimations de besoins futurs. Leurs décisions seront imparfaites, mais nécessaires.

Se fondant probablement sur un mélange de modèles rétrospectifs et prospectifs, elles devront tenir compte non seulement des facteurs habituels, comme la croissance et le vieillissement de la population et les différences régionales, mais aussi des changements survenus à l'intérieur des professions. De solides preuves démontrent qu'au cours de la dernière décennie, les médecins sont devenus de plus en plus insatisfaits de l'exercice de la médecine. Même si les candidatures aux facultés de médecine demeurent nombreuses, si la perception de l'insatisfaction est réelle et continue de prendre de l'ampleur, il pourrait devenir plus difficile d'attirer dans la profession de jeunes hommes et femmes exceptionnels.

Des preuves indiquent déjà que la médecine devient moins attrayante. Les candidatures aux facultés de médecine ne suivent pas l'augmentation du nombre de places, qui a grimpé de $31 \%$ entre 1999 et 2002, tandis que le nombre des demandes augmentait de 2,7\% seulement et le taux d'acceptation (proportion des candidats acceptés) grimpait de $28 \%{ }^{8}$. Il faut se méfier des plans qui visent à augmenter le nombre de places pour les étudiants en médecine sans s'attaquer adéquatement aux problèmes qui soustendent l'insatisfaction croissante. Il faut améliorer les conditions de travail et, dans le cas de certaines spécialités (et en particulier celles des soins primaires), les rétributions financières.

Reconnaissant les dangers posés par la prévision du nombre des médecins, $M$. Romanow a affirmé qu'il était préférable de s'en tenir à la planification à court terme et que - comme les professionnels se déplacent facilement d'une province à l'autre (et à l'étranger) - de le faire à l'échelon national par l'entremise du Conseil de la santé du Canada. L'idée n'est pas mauvaise. $-7 A M C$

\section{Références}

1. Parti libéral du Canada. Choose a health care fix for a generation [annonce]. Globe and Mail [Toronto] 19 mai 2004;A:5.

2. Barer ML, Stoddart GL. Toward integrated medical resource policies for Canada. Prepared for the Federal/Provincial/Territorial Conference of Deputy Ministers of Health, 1991.

3. Barer ML, Stoddart GL. Toward integrated medical resource policies for Canada: 1. Background, process and perceived problems. $7 A M C$ 1992;146(3): 347-51.

4. Ryten E, Thurber AD, Buske L. The Class of 1989 and physician supply in Canada. 7AMC 1998;158(6):723-8.

5. Evans RG. New bottles, same old wine: right and wrong on physician supply. 7AMC 1998;158(6):757-9.

6. Cooper RA, Getzen TE, Laud P. Economic expansion is a major determinant of physician supply and utilization. Health Serv Res 2003;38:675-96.

7. Romanow RJ. Guidé par nos valeurs : L'avenir des soins de santé au Canada. Saskatoon: Commission sur l'avenir des soins de santé au Canada; 2002. Disponible: www.commissionsoinsdesante.ca (consulté le 1 juin 2004).

8. L'Association des facultés de médecine du Canada. Conditions d'admission des facultés de médecine du Canada: admission en 2004. Ottawa; 2004. Disponible: www.acmc.ca/2004AdmissionReq/2004AdBk.pdf (consulté le 1 juin 2004). 